\title{
Adaptation of Grover's Quantum Algorithm to Multiuser Detection in an OCDMA System
}

\author{
Muhammad Idham Habibie \\ Université de Lyon, INSA Lyon, INRIA \\ CITI EA 3720 \\ F-69621 Villeurbanne, France \\ muhammad-idham.habibie@insa-lyon.fr
}

\author{
Jihad Hamie \\ Université de Lyon, INSA Lyon, INRIA \\ CITI EA 3720 \\ F-69621 Villeurbanne, France \\ jihad.hamie@insa-lyon.fr
}

\author{
Claire Goursaud \\ Université de Lyon, INSA Lyon, INRIA \\ CITI EA 3720 \\ F-69621 Villeurbanne, France \\ claire.goursaud@insa-lyon.fr
}

\begin{abstract}
To support multiple transmissions in an optical fiber, several techniques have been studied such as Optical Code Division Multiple Access (OCDMA). In particular, the incoherent OCDMA systems are appreciated for their simplicity and reduced cost. However, they suffer from Multiple Access Interference (MAI), which degrades the performances. In order to cope with this MAI, several detectors have been studied. Among them, the Maximum Likelihood (ML) detector is the optimal one but it suffers from high complexity as all possibilities have to be tested prior to decision. However, thanks to the recent quantum computing advances, the complexity problem can be circumvented. Indeed, quantum algorithms, such as Grover, exploit the superposition states in the quantum domain to accelerate the computation. Thus, in this paper, we propose to adapt the quantum Grover's algorithm in the context of MUD, in an OCDMA system using non-orthogonal codes. We propose a way to adapt the received noisy signal to the constraints defined by Grover's algorithm. We further evaluate the probability of success in detecting the active users for different noise levels. Aside from the complexity reduction, simulations show that our proposal has a high probability of detection when the received signal is not highly altered. We show the benefits of our proposal compared to the classical and the optimal ML detector.

Index Terms-MAI, ML detector, MUD, OCDMA, quantum algorithms, Grover's algorithm.
\end{abstract}

\section{INTRODUCTION}

Optical fiber transmission is a key component in current communications systems. Indeed, the ever growing number of subscribers and demand for reliable access to high-speed and high-quality services is now a very important challenge which can be addressed by exploiting the high bandwidth offered by optical fibers [1].

Nonetheless, sharing this resource requires adapted access techniques. Compared to the historical ones (Time Division Multiple Access (TDMA), Frequency DMA (FDMA), and Wavelength DMA (WDMA)), the more recent Optical Code Division Multiple Access (OCDMA) has gained significant attention from the research community during the two last decades [2]. In OCDMA systems a spreading code (or a set of spreading codes) is assigned to each potential user in the network. Whenever a user is willing to transmit, it emits its code (or one of its codes). This approach makes an

This work has been funded by AEx project QAMUT granted by INRIA France efficient use of the bandwidth as several users are simultaneously transmitting; while ensuring data confidentiality and low interception probability (thanks to the code which hides the transmitted information) and effective network control design (as the access to the medium can be done on the fly). It also permits to handle bursty traffic, and differentiated QoS and data rates [3].

OCDMA systems can be divided into two categories: coherent and non coherent schemes. In the first category, codes are bipolar (built with either $(-1)$ or $(+1)$ ) which yields a perfect orthogonality between the codes. On the contrary, incoherent OCDMA systems consider unipolar codes ((0) and (1)) [4] The latter permits the use of a simpler emitter and receiver, but at the cost of interference between simultaneously transmitted sequences. This interference is called MAI (Multiple Access Interference), and is one of the main limitations for OCDMA performance and capacity.

Numerous works have proposed code families which reduce or remove this interference. In particular, Spectral amplitude coding (SAC) approach (with numerous practical codes [5]) has gained attention thanks to its capability to entirely cancel MAI. The main drawback of this family is that it does support a large number of users due to its construction and properties.

On the other side, to use non-orthogonal codes, processing techniques have been studied in order to reduce the MAI impact on the final performance. These Multi User Detection (MUD) techniques, such as Serial Interference Cancellation (SIC) [6], and Parallel Interference Cancellation (PIC) [7], along with Hard Limiter $(H L)$ iteratively remove the users' contributions. Performance are improved, but do not reach that of the optimum Maximum Likelihood (ML) detector. The main limitation for the use of this optimal receiver is its complexity. Indeed, it tests all possible sets of user activity, which quickly becomes intractable with classical processors when the number of users increases. Fortunately, quantum computing is quickly evolving and is a promising solution to mitigate this limitation. Indeed, quantum algorithms, such as Grover, exploit the superposition states in the quantum domain to accelerate the computation.

Several papers have studied quantum multi-user detection $(Q M U D)$, using Boyer-Brassard-Høyer-Tapp $(B B H T)$ and Dürr-Høyer Algorithm (DHA) which have proven effective to 
find random values in databases with a minimum and maximum complexity of $4.5 \sqrt{N}$ and $22.5 \sqrt{N}$ [8] [9] [10], where $N$ is the database size. Similarly, [11] proposed quantum annealing for multi-user detection which helps to overcome the problem in the Non-Orthogonal Multiple Access (NOMA) receiver with Successive Interference Cancellation (SIC). In terms of Multi-Carrier CDMA (MC-CDMA), a quantum particle swarm optimization (QPSO) is an effective algorithm for multi-user detection when compared with the classical one [12].

However, to the best of the authors knowledge, the approach of using quantum algorithms for OCDMA has not been considered yet. Thus, in this paper, we propose and evaluate the benefits of using a quantum algorithm to perform MUD, in an OCDMA system using non-orthogonal codes. To do so, we adapt the Grover circuit to this problem.

This paper is structured as follows: Section II presents an overview on quantum computation, where the Grover's algorithm and Grover's circuits are described. Section III describes our adaptation of the Grover's algorithm for MUD purposes. Section $I V$ presents the performance results, based on a simple but representative system. Finally, Section $V$ concludes the paper.

\section{OVERVIEW ON THE QUANTUM COMPUTATION}

\section{A. Quantum Principle}

Inspired by quantum mechanics and benefiting from the superposition property, several problems have been solved based on quantum computation, where the complexity can be largely reduced. For example, quantum has permitted to decrease the complexity of finding the prime factor of a given integer value $n$ from $e^{\left(c(\log n)^{1} / 3(\log \log n)^{2} / 3\right)}$ to $O\left((\log n)^{2}(\log \log n)(\log \log \log n)\right)$, where $c$ is a constant in the classical case [13]. Furthermore, quantum permits to search a single value in a large unsorted database of size $N$ with a complexity of $O(\sqrt{N})$ (instead of $O(N)$ in a classical case). Moreover, it is proved that there are some problems, which cannot be solved using the classical computation (e.g. Boolean Satisfiability SAT problem) but, can be solved using quantum computation techniques [14] [15]. Indeed, thanks to the superposition, several simultaneous tasks are performed and thus, the requested number of computations can be highly reduced.

As a matter of fact, the quantum accelerates the computation using a new type of bits, which are so-called as quantum bits (qubits). The qubits are able to be in the state 0 and 1 simultaneously in a superposed state [16]. It is denoted with the Dirac notation $|$.$\rangle . The notation of a qubit |\psi\rangle$ is written as follows :

$$
|\psi\rangle=\alpha|0\rangle+\beta|1\rangle
$$

where $\alpha$ and $\beta$ are normalized complex numbers, which verifies $|\alpha|^{2}+|\beta|^{2}=1$. The superposed state is only possible within the quantum processor and can not be accessed outside. However, it is possible to make a measurement on the qubit. This will randomly project the qubit on $|0\rangle$ or $|1\rangle$ with respect

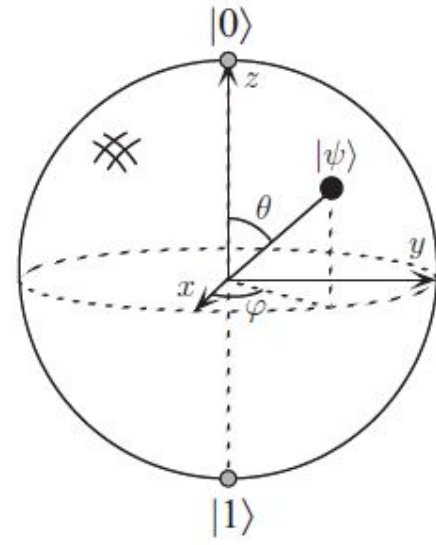

Fig. 1. Bloch Sphere [17]

to their associated probabilities. More precisely, $|\alpha|^{2}$ and $|\beta|^{2}$ are respectively the probabilities of being in state $|0\rangle$ and $|1\rangle$. If $\alpha=0 ; \beta=1$, then $|\psi\rangle=1$. In contrast, $\beta=0$ and $\alpha=1$ always returns $|\psi\rangle=0$. In addition, the superposition state where $\alpha=\frac{1}{\sqrt{2}}$ and $\beta=\frac{1}{\sqrt{2}}$ leads to $50 \%$ probability of having 0 or 1 .

Since $\alpha$ and $\beta$ are normalized complex numbers, it is possible to transform eq.(1) into:

$$
|\psi\rangle=\cos \frac{\theta}{2}|0\rangle+e^{i \varphi} \sin \frac{\theta}{2}|1\rangle
$$

where $0 \leqslant \theta \leqslant \pi$ and $0 \leqslant \varphi \leqslant 2 \pi$. Hence, it is then possible to illustrate $|\psi\rangle$ in a unit sphere as shown in Fig.1 [17].

\section{B. Grover Algorithm}

Let's consider an unsorted database of size $N$. Grover's algorithm objective is to retrieve within the database the index of the element verifying a given constraint. If we denote by $f$ the function which permits to relate the index $x$ to the register value, Grover's algorithm solves $f(x)=\delta$, where $\delta$ is the desired value. To do so, Grover's algorithm consists of two parts ; Oracle and Diffuser as shown in Fig. 2. The Oracle marks the states which verify the targeted constraints. The related operation is defined as follows:

$$
U_{w}|q\rangle=(-1)^{f(x)}|q\rangle= \begin{cases}U_{w}|q\rangle & f(x) \neq \delta \\ -U_{w}|q\rangle & f(x)=\delta\end{cases}
$$

where $\delta$ denotes the desired value, $f(x)$ is the Grover function. If $f(x)$ is equal to $\delta$, the oracle changes the associated amplitude to a negative one.

Then, the marked qubit is amplified using diffuser, which is denoted as follows:

$$
U_{s}|q\rangle=2|s\rangle\langle s|-I
$$

Towards finding the desired solutions, the Oracle and Diffuser are performing several iterations. This number of iterations is in the order of $O(\sqrt{N})$ or less [16]. In this context, [16] has defined and expressed the optimum number 


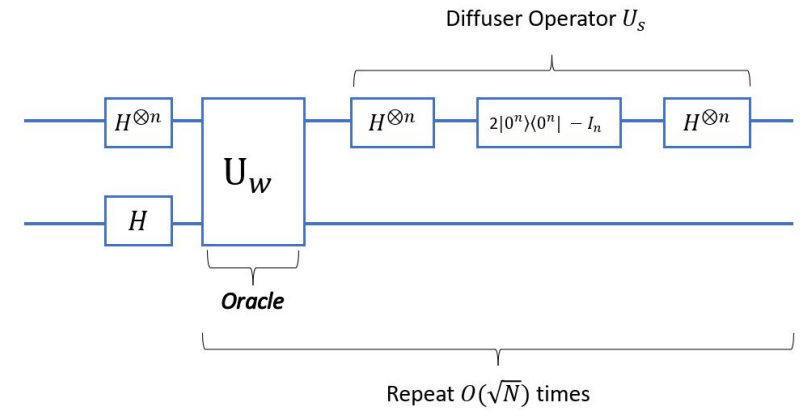

Fig. 2. Grover Scheme

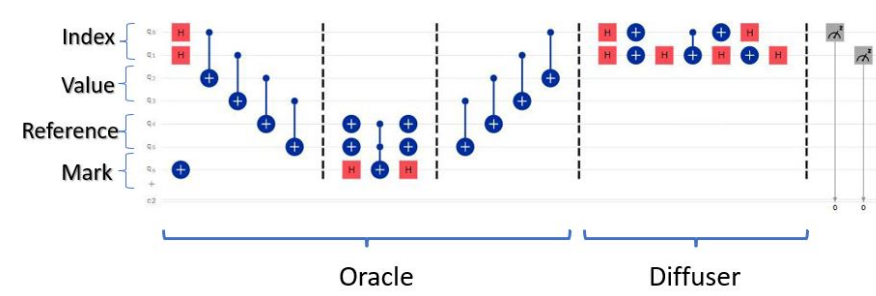

Fig. 3. General Grover Circuit of 2 qubits.

of iterations in order to find a specific solution. This optimal number is depending on the size $N$ of the involved database and on the existing number of solutions, which is denoted as $S$. The optimal number of iterations is given as follows:

$$
L_{o p t}=\lfloor\pi / 4 \sqrt{N / S}\rfloor
$$

After $N_{I}$ iteration steps, the probability of success $\left(P_{s}\right)$ is given by [18]:

$$
P_{s}\left(N_{I}\right)=\sin ^{2}\left(\left(2 N_{I}+1\right) \theta_{s}\right)
$$

where $\sin ^{2} \theta_{s}=\frac{S}{N}$.

\section{Grover Circuit Implementation}

The Grover circuit design can be simulated using Qiskit library provided by Python. Qiskit provides numerous advantages to build and analyse the quantum gates in order to implement the quantum algorithm. The Grover circuit relies on four different registers: 1) Index register 2) Value register 3) Reference register 4) Mark register [19]. The Index register contains the argument of the function $f(x)$ and will provide the delivered solution at the end of the algorithm. The Value Register contains the value of the function $f(x)$ (in eq.3) applied into the index register state, whereas Reference Register corresponds to the desired value $(\delta)$. The Mark register provides the negative sign into the computation, to mark the valid states. Figure 3 illustrates a basic two qubits Grover circuit, where all the four different registers are illustrated. In such settings, the mark and diffusion part are the same, only adapted to the size of the considered problem. However, the part related to the function is specific to the problem.

\section{PROPOSED SYSTEM}

\section{A. System Model}

In this paper, we propose to adapt Grover's algorithm to perform the Multi-User Detection in an OCDMA system, and we evaluate the performance.

We consider a network where each user is assigned a unique code, and transmits the code when sending a bit $b_{i}=1$, while it remains silent for a 0 . The users' signals are propagated over the same fiber and are observed at the receiver side as a summation of all transmitted contributions. It must be noted that the transmission is classical (only the decoding will be performed in quantum domain). In addition, we consider a transmission model where all emitted signals arrive simultaneously at the receiver with the same amplitude (thanks to a feedback loop for example). We model the channel as a perfect one with gain $h=1$, along with an Additive White Gaussian Noise (AWGN) denoted by $n$. Thus, the received signal can be written as follows:

$$
y=\sum_{i=1}^{K} b_{i} c_{i}+n
$$

where $K$ denotes the number of users, $i$ is the index of a specific user, $b_{i} \in\{0,1\}$ is the bit transmitted by user $i, c_{i}$ is the corresponding codeword and $n$ is the Gaussian noise following $\mathcal{N}\left(\mu, \sigma^{2}\right)$.

The objective of the receiver is to determine the values of all the transmitted bits $\hat{b}=\left\{\hat{b}_{i} \mid i \in\{1, \ldots, K\}\right\}$. To do so, each user can be treated separately, but this leads to a high error probability. On the contrary, considering all users jointly leads to a better accuracy. In particular, when all combinations can be observed with the same probability, it is well known that the optimum multi-user detector is the ML (Maximum Likelihood) one [20]. This detector identifies the most likely transmitted bits set given the knowledge of the received signal, as in :

$$
\hat{b}=\underset{b}{\operatorname{argmax}}(\mathbb{P}(Y \mid b))
$$

The ML provides the best accuracy, but at the cost of a high complexity as all possible combinations for $b=b_{i}, i \in$ $\{0,1, \ldots, K\}$ have to be evaluated. However, we can observe that as $b_{i} \in\{0,1\}$, we can exploit the superposition property of qubit by assigning one qubit to each user transmitted bit. This permits to evaluate simultaneously all possibilities and select the wanted one.

\section{B. Proposed quantum algorithm}

In a noiseless case, the received signal $y$ is a vector of size $S F$ containing only integer values. It can be fed into quantum algorithm after quantification. The binary size for each component depends on the highest value which can be obtained in the corresponding slot.

However, when noise is taken into account, $y$ has real components. But, Grover's algorithm must only be fed by binary numbers, and will search for the $b$ set which can create this signature. A simple and accurate way to overcome this 
issue is obtained by approximating the $y$ to the closest integer values. Thus, Grover's algorithm is fed with $\hat{y}$, which is given by:

$$
\hat{y}=\max (0, \operatorname{round}(y))
$$

All these previous operations are performed with classical devices as the computation remains simple. The processing in the quantum domain starts with the appropriate Grover algorithm which is then applied. The algorithm is run with the optimum number of iterations, and provides as an output the set of the estimated transmitted bits.

The proposed algorithm can be summarized as follows:

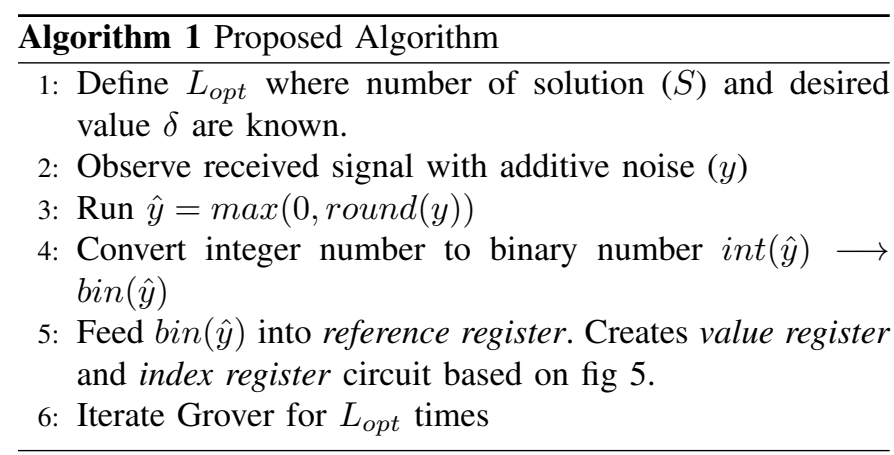

\section{Simulation Setup And Results}

\section{A. Simulation setup}

To validate our approach, we have considered an example. We have been constrained to use a reduced network configuration, in order to be able to use the quantum simulation tools. Thus, we have focused on codes with a small spreading factor. We have empirically selected codes that permit to handle the maximum number of users, such that any 2 different subsets of the code do not lead to the same superposed signature. With this constraint, we thus ensure that the function which relates the activity set $b$ to the received signal $y$ is injective, therefore that, in a noiseless case, the error probability is null.

In this work, we have considered $S F=3$. We have selected 4 codewords as follows; $c_{1}=[1,1,0], c_{2}=[0,1,1], c_{3}=$ $[0,0,1], c_{4}=[1,0,1]$.

Based on these codes, the oracle function, which evaluates the expected received signal as a function of the transmitted bits has been implemented in Qiskit, leading to the circuit presented on Fig. 4. We can note that two qbits are used to represent each $\hat{y}$ components. This is due to the fact that for the considered codeset, the maximum value that can be reached on any slot is 3 .

\section{B. Noiseless case performances}

To evaluate the performance, we first consider the ideal case where no noise impacts the received signal. In this case, for the classical ML algorithm, the success probability is $P_{s}=1$.

As for the Grover algorithm, we have plotted in Fig. 5, the probabilities of recovering any set, when the actual transmitted set is $\{0,1,0,0\}$, after running the Grover's algorithm with $L_{\text {opt }}=3$ iterations and under noiseless channel. We can

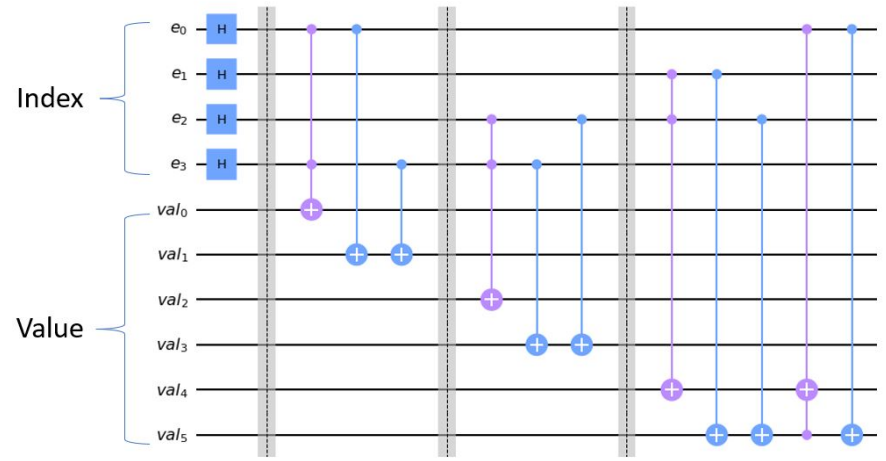

Fig. 4. Grover Circuit Function.

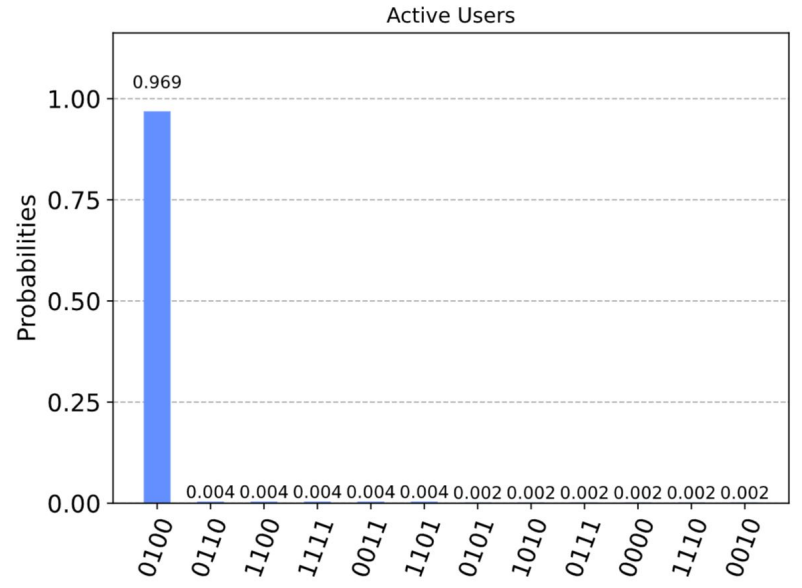

Fig. 5. Measurement statistics after running the Grover's algorithm for $L_{\text {opt }}=3$ iterations, under noiseless channel

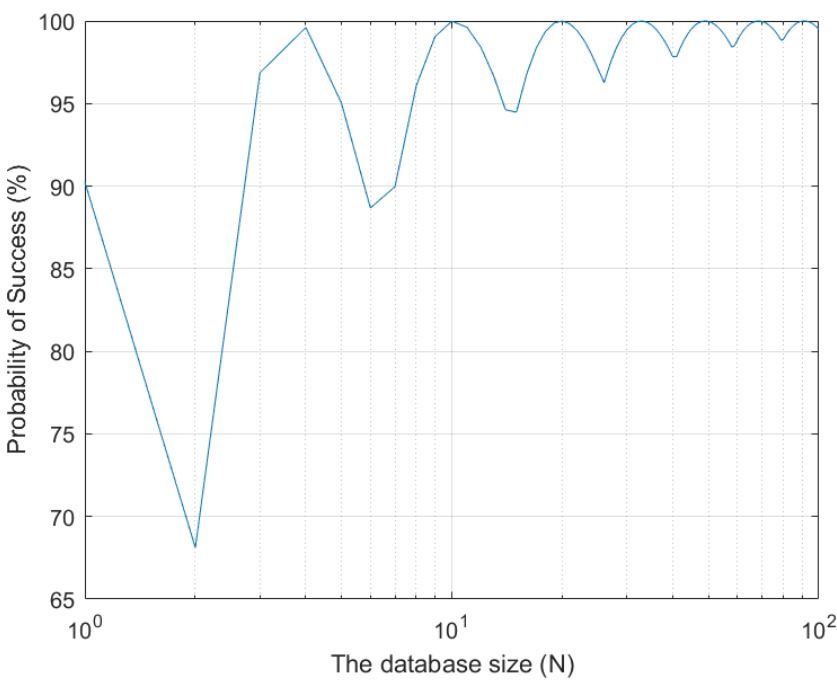

Fig. 6. Quantum success probability (in \%) as a function of the database size $N$. 
observe that the Grover's delivers the correct solution with a probability of detection of $96.9 \%$. This is due to the fact that for a small dataset size, the cancellation of the non desired states is not possible in a small number of iterations.

On the contrary, we can see on Fig. 6 that as the database size increases, the success probability tends to 1 . Thus, the quantum algorithm efficiency increases for larger number of users.

\section{Noisy case performances}

In this part, we take the noise into account. It follows a normal law with mean $\mu=0$ and a standard deviation denoted by $\sigma$. We can encounter two situations.

In the first situation, the noise does not lead to a modification of the signature $\hat{y}$ fed into Grover's algorithm. Indeed, the noise contribution is small enough and is removed by the rounding function applied to the received signal $y$. Thus, the quantum computation has a high success probability to correctly identify the users, and presents the same performances than in the noiseless case. This is often the case for small noise standard deviation.

In the second situation, with larger noise deviation, the signature $\hat{y}$ is modified; either as another valid signature (leading to an almost null success probability), or a nonvalid one (leading to equiprobable statistics among all the $(N)$ possible solutions in the database).

In practice, the system faces the two situations, with different balancing, depending on the actual noise deviation. The success probability is thus obtained with a linear combination of the 2 situations.

The optimal ML detector and our proposed algorithms are compared in Fig. 7. This figure shows the variation of the average probability of success in detecting the active users, as a function of the noise standard deviation $\sigma$. For each noise variance, 400 independent noise process realizations are tested, and averaged. We can first note that the performance are degraded when the noise's variance increases. The success probability goes from the maximum one in the noiseless case $(96 \%)$, to a random selection with equiprobable variables $(\%=100 / 16)$. Besides, as expected, the ML provides the best accuracy as it is the optimal algorithm, but one may note that our proposal gives results that are comparable with the ML detector and more particularly when the noise level is relatively small. Moreover, thanks to Fig. 6, we can expect the performance difference to be decreased for larger network size, as Grover's algorithm become more accurate. In addition, our quantum proposal outperforms the ML detector in terms of complexity, as here the ML detector requires 16 evaluation, while Grover's reads only. This benefit increases with the number of users. We can thus observe that our proposed solution permits to significantly reduce the computation delay at the cost of a reasonable impact on the performance. Moreover, our proposal can be a promising detector of active users in a massive communication networks.

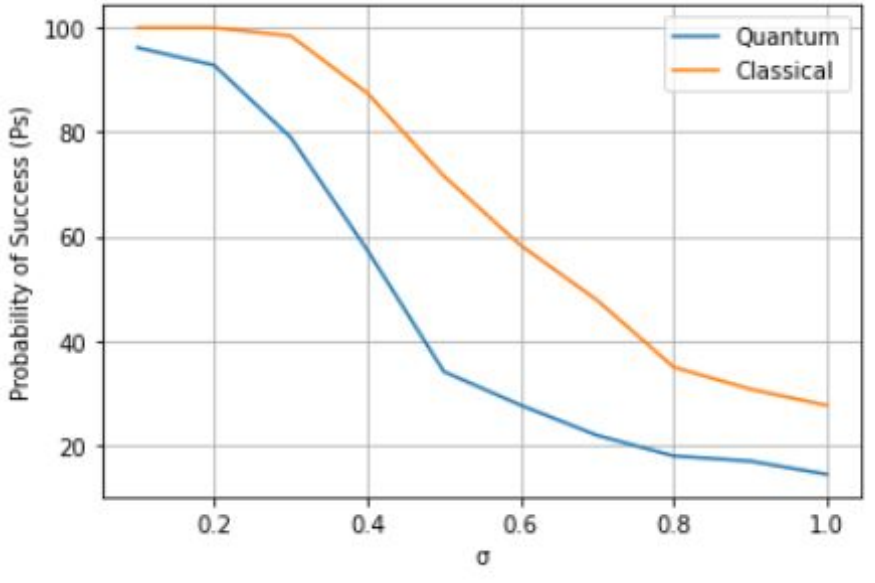

Fig. 7. Probability of Success as a function of Noise Variance

\section{CONCLUSION}

In this paper, we have adapted the Grover's quantum algorithm for MUD purposes in an OCDMA communication scheme. The adapted Grover's algorithm is fed by noisy received signals, which are preliminary reshaped in order to be compliant with the Grover's algorithm constraints. The probability of detection of active users has been assessed as a function of SNR (or noise variance). The obtained results on a small network size have shown that our proposal has comparable detection performance with the optimum ML detector, but with a lower complexity. Interestingly, results have proved that as the database size increases, the success probability tends to 1 (in a noiseless case). Thus, we conclude that the quantum algorithm will be more pertinent for a large number of users.

\section{REFERENCES}

[1] Alaan Ghazi et al. "Performance Analysis of ZCCOptical-CDMA over SMF for Fiber-To-The-Home Access Network". In: Journal of Physics: Conference Series 1529.2 (2020). ISSN: 17426596. DOI: 10.1088/ 17426596/1529/2/022013.

[2] Hichem Mrabet et al. "A Comparative Study of Asynchronous and Synchronous OCDMA Systems". In: IEEE Systems Journal 15.3 (2020), pp. 3642-3653. ISSN: 1932-8184. DOI: 10.1109/jsyst.2020.2991678.

[3] Majid H. Kakaee et al. "Development of Multi-Service (MS) for SACOCDMA systems". In: Optics and Laser Technology 60 (2014), pp. 49-55. ISSN: 00303992. DOI: 10.1016/j.optlastec.2014.01.002. URL: http://dx.doi.org/ 10.1016/j.optlastec.2014.01.002.

[4] Chih Ta Yen and Chih Ming Chen. "A study of three-dimensional optical code-division multiple-access for optical fiber sensor networks". In: Computers and Electrical Engineering 49 (2016), pp. 136-145. ISSN: 00457906. DOI: 10.1016/j.compeleceng.2015.02.016. URL: http://dx.doi.org/10.1016/j.compeleceng.2015.02. 016.

[5] Soma Kumawat and Ravi Maddila. "A Review on Code Families for SAC-OCDMA Systems". In: (Jan. 2020), pp. 307-315. DOI: 10.1007/978-981-13-6159-3 33

[6] Claire Goursaud et al. "Serial Interference Cancellation receiver for Optical CDMA systems". In: Proc.of 5th IEEE International Conference on ITS (Intelligent Transportation System) Telecommunications, Brest (June 2005), pp. 403-406.

[7] Claire Goursaud et al. "DS-OCDMA receivers based on Parallel Interference Cancellation and Hard-Limiter". In: IEEE Transactions on Communications 54 (Sept. 2006), pp. 1663-1671. DOI: 10.1109/TCOMM.2006. 881252. 
[8] Panagiotis Botsinis, Soon Xin Ng, and Lajos Hanzo. "Quantum search algorithms, quantum wireless, and a low-complexity maximum likelihood iterative quantum multi-user detector design". In: IEEE Access 1 (2013), pp. 94-122. ISSN: 21693536. DOI: 10.1109/ACCESS. 2013.2259536.

[9] Panagiotis Botsinis et al. "Fixed-Complexity QuantumAssisted". In: 62.3 (2014), pp. 990-1000.

[10] Panagiotis Botsinis et al. "Iterative quantum-assisted multi-user detection for multi-carrier interleave division multiple access systems". In: IEEE Transactions on Communications 63.10 (2015), pp. 3713-3727. ISSN: 00906778. DOI: 10.1109/TCOMM.2015.2458857.

[11] Refik Caglar Kizilirmak. "Quantum Annealing Approach to NOMA Signal Detection". In: 2020 12th International Symposium on Communication Systems, Networks and Digital Signal Processing, CSNDSP 2020 (2020), pp. 1-5. DOI: 10. 1109/CSNDSP49049. 2020. 9249501.

[12] Hongyuan Gao and Ming Diao. "Quantum particle swarm optimization for MC-CDMA multiuser detection”. In: 2009 International Conference on Artificial Intelligence and Computational Intelligence, AICI 20092 (2009), pp. 132-136. DOI: 10.1109/AICI.2009.469.

[13] Peter W. Shor. "Polynomial-time algorithms for prime factorization and discrete logarithms on a quantum computer". In: SIAM Journal on Computing 26.5 (1997), pp. 1484-1509. ISSN: 00975397. DOI: 10 . 1137 / S0097539795293172. arXiv: 9508027 [quant-ph].

[14] Juexiao Su, Tianheng Tu, and Lei He. "A quantum annealing approach for boolean satisfiability problem". In: Proceedings - Design Automation Conference 05- 09-June-2016 (2016), pp. 2-7. ISSN: 0738100X. DOI: $10.1145 / 2897937.2897973$

[15] Carlos Barron-Romero. "Classical and Quantum Algo- ' rithms for the Boolean Satisfiability Problem". In: 180 (2015), arXiv: 1510.02682. URL: http://arxiv.org/abs/ 1510.02682.

[16] Manuel Vogel. Quantum Computation and Quantum Information, by M.A. Nielsen and I.L. Chuang. Vol. 52.6. 2011, pp. 604-605. ISBN: 9781107002173. DOI: 10. 1080/00107514.2011.587535.

[17] Ian Glendinning. The Bloch Sphere. Tech. rep. 2005, pp. 63-82.

[18] Ahmed Younes. "Strength and weakness in grover's quantum search algorithm". In: arXiv preprint arXiv:0811.4481 (2008).

[19] Panagiotis Botsinis et al. "Quantum Error Correction Protects Quantum Search Algorithms Against Decoherence". In: Scientific Reports 6.November (2016), pp. 1-13. ISSN: 20452322. DOI: 10. 1038/ srep38095. URL: http://dx.doi.org/10.1038/srep38095.

[20] Sergio Verdu et al. Multiuser detection. Cambridge university press, 1998. 